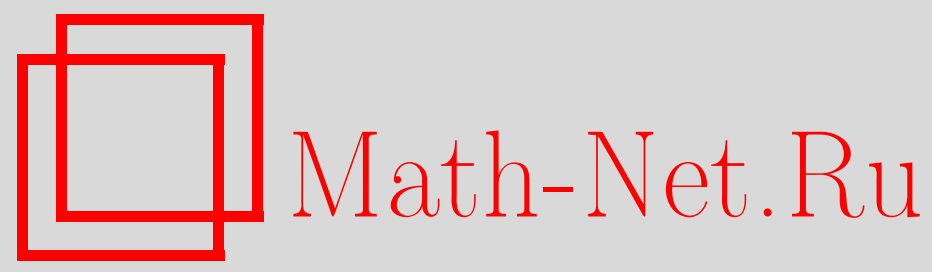

А. Н. Старков, Тихоновское свойство для линейных групп, Матем. заметки, 1998, том 63, выпуск 2, 269-279

DOI: https://doi.org/10.4213/mzm1273

Использование Общероссийского математического портала Math-Net.Ru подразумевает, что вы прочитали и согласны с пользовательским соглашением http://www. mathnet.ru/rus/agreement

Параметры загрузки:

IP: 3.93.64.190

26 апреля 2023 г., 05:47:13

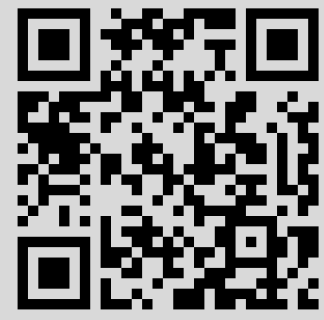




\title{
ТИХОНОВСКОЕ СВОЙСТВО ДЛЯ ЛИНЕЙНЫХ ГРУПП
}

\author{
А. Н. Старков
}

В работе доказан критерий тихоновости для широкого класса топологических групп, включающих линейные дискретные группы и группы Ли. Главный результат дает критерий тихоновости для почти полициклических групп. Ввиду известной альтернативы Титса это дает требуемый критерий для линейных дискретных групп. В заключение отмечено, что частный случай приведенного доказательства дает критерий тихоновости для групп Ли. Кроме того, построен пример полицик лической группы, которая не имеет тихоновских подгрупп конечного индекса.

Библиограффия: 7 названий.

Введение. В этой работе мы доказываем критерий тихоновости для широкого класса топологических групш. Этот класс включает линейные дискретные группы и группы Ли. Определение тихоновской группы было введено Фюрстенбергом [1] в ходе изучения случайньх блужданий на группах. Он, в частности, доказал, что нильпотентные группы Ли и параболические подгрупшы в полупростых группах Ли являются тихоновскими.

Изучение тихоновского свойства для локально компактных групп было продолжено Конще и Гиваршем [2]. Они доказали критерий тихоновости для групп Ли и построили пример тихоновской групш Ли с нетихоновской подгруппой Ли. Кроме того, они доказали, что если подгруппа $F \subset G$ равномерна (т.е. факторпространство $G / F$ компактно) в локально компактной тихоновской групше, то она также является тихоновской.

Недавно Григорчук [3] получил ряд результатов о тихоновских свойствах счетных групп. Основной из них состоит в том, что конечно порожденная бесконечная тихоновская группа допускает эпиморфизм на аддитивную группу $\mathbb{Z}$ целых чисел.

Наш главньй результат дает критерий тихоновости для почти полициклических групп; его доказательство достаточно элементарно и не использует теорию случайных блужданий. Ввиду известной альтернативы Титса [4] это дает требуемьй критерий для линейных дискретных групп. В заключение мы отмечаем, что частный случай нашего доказательства дает критерий тихоновости для групп Ли. Кроме того, мы строим пример полициклической групшы, которая не имеет тихоновских подгрупп конечного индекса.

1. Определения и соглашения. Вначале изложим подготовительный материал. Запись $G=F \cdot H$ будет означать, что группа $G$ есть полупрямое произведение подгруппы $F \subset G$ и нормальной подгруппы $H \subset G$, т.е. $G=F H$ и $F \cap H=1$, где $1-$

Работа выполнена при поддержке Российского фонда фундаментальных исследований, грант № 96-01-00182, и фонда Американского математического общества. 
единичньй элемент в $G$. Если $F$ - подгруппа в топологической группе $G$, то $\bar{F}$ обозначает замькание $F$ в топологии групшы $G$. Далее $[g, h]=g h g^{-1} h^{-1}$ обозначает коммутатор элементов $g, h \in G$, а $Z_{G}(H)$ - централизатор подгрупшы $H \subset G$ в $G ; \mathbb{Z}(a) \subset G$ и $\mathbb{Z}_{n}(b) \subset G$ - соответственно свободная циклическая группа и группа порядка $n$, порожденные элементами $a, b \in G ; \mathbb{R}^{*}$ - мультипликативная группа положительных чисел; $\mathbb{R}^{+}$- замкнутое множество всех неотрицательных чисел. Абелева группа непрерьвных мультипликативных характеров $\chi: G \rightarrow \mathbb{R}^{*}$ обозначается через $X^{+}(G)$, векторное пространство непрерьвных аддитивных характеров - через $X(G)$. Ясно, что отображение $\log : X^{+}(G) \rightarrow X(G)$ является изоморфизмом.

ОПРЕДЕЛЕНИЕ 1. Пусть $L$ - локально вьпуклое линейное топологическоепространство и $\Phi: L \rightarrow \mathbb{R}$ - линейный непрерывный функционал. Тогда $K \subset L$ есть выпуклый конус с компактной базой, если $B=K \cap \Phi^{-1}(1)$ является вьпуклым компактом и $K=\mathbb{R}^{+} B$.

ОПРЕДЕЛЕНИЕ 2. Топологическая групша $G$ назьвается тихоновской, если для любого линейного непрерывного действия на локально вьпуклом линейном топологическом пространстве $L$ в каждом $G$-инвариантном конусе $K \subset L$ с компактной базой найдется $G$-инвариантньй луч $\mathbb{R}^{+} x \subset K$.

ЗАмЕчАниЕ. Такие действия естественно возникают в теории случайных блужданий. Пусть, например, $G$ - счетная группа и $\mu$ - вероятностная мера на $G$. Можно определить правое случайное блуждание на $G$ следующим образом: за время $\Delta t=1$ мы перемешаемся из точки $g \in G$ в точку $g h \in G$ с вероятностью $\mu(h)$. Определим марковский оператор $P$ на пространстве $L^{1}(G, \mu)$ формулой

$$
P f(g)=\sum_{h \in G} \mu(h) f(g h) .
$$

Если в данньй момент мы находимся в точке $g \in G$, то $P f(g)$ есть математическое ожидание функции $f$ в следующий момент времени. Функция $f$ на $G$ назьвается $\lambda$-гармонической, если $P f=\lambda f$, и $\lambda$-субгармонической, если $P f \leqslant \lambda f$. Можно проверить, что множество положительных $\lambda$-субгармонических функций образует выпукльй конус с компактной базой. Если $\mu$ имеет конечньй носитель, то конус положительных $\lambda$-гармонических функций также имеет компактную базу.

Заметим, что факторгрупша тихоновской групшы обязана быть тихоновской. Более того, если $p: G \rightarrow G^{\prime}$ - непрерывный гомоморфизм топологических групп такой, что образ $p(G)$ плотен в $G^{\prime}$, и $G$ является тихоновской, то и $G^{\prime}$ является тихоновской.

Если группа $G$ конечна, то барицентр любой орбиты $G(x) \subset K G$-неподвижен. Поэтому конечная группа всегда обладает даже неподвижным лучом (в частности, является тихоновской).

Одно из эквивалентных определений аменабельной группы состоит в том, что для аффинного действия $G$ на локально выпуклом линейном топологическом пространстве $L$ в каждом $G$-инвариантном выпуклом компакте $B \subset L$ найдется $G$-неподвижная точка $x \in B$. Если мы имеем линейное непрерьвное действие $G$ на конусе $K$, то на его базе $B$ можно определить непрерывное действие $G$ формулой

$$
\widetilde{g}(x)=\frac{g(x)}{\Phi(g(x))}
$$


(заметим, что из $g(K)=K$ вытекает $g(0)=0$, поэтому действие $G$ на $B$ определено корректно). По теореме Шаудера-Тихонова [5] гомеоморфизм выпуклого компакта всегда имеет неподвижную точку, поэтому циклическая группа является тихоновской. Однако, индуцированное действие $G$ на $B$ не обязано быть аффинным и поэтому аменабельная группа, вообще говоря, не обязана быть тихоновской.

Простейшим примером нетихоновской группы (см. [1]) является полупрямое произведение

$$
G=\mathbb{Z}_{2}(\sigma) \cdot \mathbb{Z}(a) \subset \mathrm{GL}(2, \mathbb{R}), \quad \text { где } \quad \sigma=\left(\begin{array}{ll}
0 & 1 \\
1 & 0
\end{array}\right), \quad a=\left(\begin{array}{cc}
2 & 0 \\
0 & 1 / 2
\end{array}\right)
$$

(конус $\{x, y \geqslant 0\}$ является $G$-инвариантным и не имеет $G$-инвариантного луча).

С другой стороны, любая тихоновская группа является аменабельной. В самом деле, пусть $G$ действует аффинно и непрерьвно на вьпуклом компакте $B$ в линейном топологическом пространстве $L$. Зададим действие $G$ на $L^{*}=L \times \mathbb{R}$ следуюшим образом. Если $g(x)=A x+b$, где $A: L \rightarrow L$ - линейньй непрерьвньй оператор и $b \in L$, то положим $g^{*}(x, t)=(A x+t b, t)$. Легко проверить, что полученное таким образом действие линейно, непрерьвно и совпадает с исходным действием на $L \times\{1\}$. Конус $K=\mathbb{R}^{+}(B, 1)$ является $G$-инвариантным и ввиду тихоновости содержит $G$-инвариантньй луч $\mathbb{R}^{+}(x, 1)$. Точка $x \in L$, очевидно, является $G$-неподвижной.

2. Тихоновское свойство для полициклических групп. Подгруппа $N$ группы $G$ назьвается нильрадикалом в $G$, если $N$ является максимальной нормальной нильпотентной подгруппой в $G$. Заметим, что даже в конечно порожденной группе нильрадикал не обязан существовать. Группа $F$ называется почти абелевой, если она обладает нормальной абелевой подгрупой $A \subset F$ конечного индекса в $F$. Группа Г называется полиииклической, если найдется конечная последовательность $1=\Gamma_{0} \subset \Gamma_{1} \subset \ldots$ $\subset \Gamma_{k}=\Gamma$ такая, что $\Gamma_{l-1}-$ нормальная подгруппа в $\Gamma_{l}$ и факторгруппа $\Gamma_{l} / \Gamma_{l-1}$ является циклической для всех $l=1, \ldots, k$. Очевидно, что каждая полициклическая группа является конечно порожденной. Всюду далее Г предполагается полициклической группой.

ОСНОВНОЙ ФАКТ О ПОЛИЦИКЛИЧЕСКИХ ГРУППАХ. Каждая полиииклическая группа Г обладает нильрадикалом ${ }^{n} \Gamma$, причем ${ }^{n}$ Г является конечно порожденной группой и факторәруппа $\Gamma /^{n} \Gamma$ является почти абелевой.

Известно (см. [3], [2] или лемму 2), что конечно порожденная нильпотентная группа является тихоновской. Поскольку факторизация сохраняет свойство тихоновости, вначале мы должны найти критерий тихоновости для почти абелевых групп. Но предварительно мы укажем широкий класс топологических груп, не являющихся тихоновскими.

Рассмотрим группу $E_{n}=\mathrm{O}(n, \mathbb{R}) \cdot \mathbb{R}^{n}$ движений евклидовой метрики на $\mathbb{R}^{n}$.

Лемма 1. Пусть $G \subset E_{n}$ - замкнутая неабелева подгруппа такая, что пересечение $L=G \cap \mathbb{R}^{n}$ является равномерной подгруппой в $\mathbb{R}^{n}$. Тогда $G$ не является тихоновской.

ДокАЗАтЕЛьСтво. Пусть $C$ - нетривиальная компактная подгруппа в $\mathrm{O}(n, \mathbb{R})$ такая, что $G \mathbb{R}^{n}=C \cdot \mathbb{R}^{n}$. Поскольку действие компактной подгруппы $C$ на $\mathbb{R}^{n}$ вполне приводимо, мы можем без ограничения общности предположить, что оно неприводимо. В самом деле, если $\mathbb{R}^{n}=\mathbb{R}^{k}+\mathbb{R}^{n-k}$ - разложение в сумму $C$-инвариантных подпространств (причем действие $C$ на $\mathbb{R}^{k}$ нетривиально), мы можем рассмотреть проекцию 
$p: C \cdot \mathbb{R}^{n} \rightarrow C^{\prime} \cdot \mathbb{R}^{k}$, где $C^{\prime} \subset \mathrm{O}(k, \mathbb{R})$ - нетривиальная компактная подгруппа, являющаяся образом $C$, и взять замыкание $G^{\prime}=\overline{p(G)}$.

Итак, не ограничивая общности, действие $C$ на $\mathbb{R}^{n}$ нетривиально и неприводимо. В таком случае группа непрерьвных аддитивных характеров $X(G)=\{\chi: G \rightarrow \mathbb{R}\}$ тривиальна. В самом деле, $\mathbb{R}^{n} \cap \operatorname{Ker}(\chi)$ является нормальной подгруппой в $G$, и, следовательно, в $C \cdot \mathbb{R}^{n}$. Поэтому ее линейная оболочка $A \subset \mathbb{R}^{n}$ является $C$-инвариантньм подпространством, и ввиду неприводимости либо $A=\mathbb{R}^{n}$, либо $A=0$. В первом случае факторгруппа $\chi(G)=G / G \cap \operatorname{Ker}(\chi)$ компактна, т.е. характер $\chi$ тривиален. Во второмиз соображений размерности $n=1$ - либо $G=\mathbb{Z}_{2} \cdot \mathbb{Z}$, либо $G=\mathbb{Z}_{2} \cdot \mathbb{R}$, и група характеров также тривиальна.

Рассмотрим теперь пространство $H$ бесконечно гладких функций на $\mathbb{R}^{n}$ с естественным представлением группы $E_{n}=\mathrm{O}(n, \mathbb{R}) \cdot \mathbb{R}^{n}$ :

$$
\left(\rho_{c} f\right)(x)=f(c(x)), \quad c \in \mathrm{O}(n, \mathbb{R}), \quad x \in \mathbb{R}^{n}, \quad\left(\rho_{a} f\right)(x)=f(x+a), \quad a, x \in \mathbb{R}^{n} .
$$

Известно [6], что $H$ является пространством $\Phi$ реше по отношению к семейству преднорм

$$
p_{k, m}(f)=\sup _{\substack{|x| \leqslant m \\ 0 \leqslant l \leqslant k}}\left\{\frac{\partial^{l} f}{\partial^{i_{1}} x_{1} \cdots \partial^{i_{n}} x_{n}}(x): i_{1}+\cdots+i_{n}=l\right\} .
$$

Известно также, что оператор Лапласа $\Delta_{n}$ на $H$ коммутирует с действием $E_{n}$. Для фиксированного $\lambda>0$ определим $f_{1}(x)=\exp \left(\sqrt{\lambda} x_{1}\right)$. Пусть $K$ есть вьпуклая оболочка орбиты $E_{n}\left(f_{1}\right)$. Так как $H$ есть пространство $\Phi$ реше, выпуклая оболочка $B$ компактной орбиты $\mathrm{O}(n, \mathbb{R})\left(f_{1}\right)$ также компактна. Ясно, что $K$ является $G$-инвариантньм конусом с компактной базой $B$ и состоит из функций, удовлетворяющих уравнению $\Delta_{n} f=\lambda f$. Однако, конус $K$ не содержит $G$-инвариантного луча: поскольку $X(G)=0$, он был бы неподвижен и состоял бы из функций $f$, инвариантньх относительно трансляций вдоль $L=G \cap \mathbb{R}^{n}$. Предположим, что $f \neq 0$. Легко видеть, что локальньй максимум решения уравнения $\Delta_{n} f=\lambda f$ неположителен, а локальньй минимум неотрицателен. Поскольку $L=G \cap \mathbb{R}^{n}$ является равномерной подгруппй в $\mathbb{R}^{n}$, функция $f$ имеет как локальные максимумы, так и локальные минимумы, и значит, тождественно равна 0.

Заметим, что если свободная абелева грушпа $\mathbb{Z}^{n}$ является нормальной подгруппой в счетной группе $G$, то можно расширить $\mathbb{Z}^{n}$ и $G$ до $\mathbb{R}^{n}$ и $A(G)$ соответственно таким образом, что $G \subset A(G), G \cap \mathbb{R}^{n}=\mathbb{Z}^{n}, G \mathbb{R}^{n}=A(G)$ и $\mathbb{R}^{n}$ - нормальная подгруппа в $A(G)$. В частности, $F=G / \mathbb{Z}^{n} \simeq A(G) / \mathbb{R}^{n}$. В таком случае будем говорить, что $A(G)$ получена из $G$ взятием линейной оболочки $\mathbb{Z}^{n} \subset G$. Группу $A(G)$ можно построить, например, следуюшим образом. Рассмотрим вложение $i: \mathbb{Z}^{n} \subset \mathbb{R}^{n}$ и зададим действие $G$ на $\mathbb{R}^{n}$, полученное из линейного продолжения действия $G$ на $\mathbb{Z}^{n}$ внутренними автоморфизмами. Возьмем теперь полупрямое произведение $G \cdot \mathbb{R}^{n}$, построенное по этому действию, и положим $A(G)=G \cdot \mathbb{R}^{n} / \Delta$, где $\Delta=\left\{(x,-i(x)): x \in \mathbb{Z}^{n}\right\}$ - нормальная подгруппа в $G \cdot \mathbb{R}^{n}$. Заметим, что если $G=\mathbb{Z}^{n}$, то $A(G)=\mathbb{R}^{n} ;$ если же $G=\frac{1}{2} \mathbb{Z}^{n}$ и $\mathbb{Z}^{n}$ рассматривается как подгруппа в $G$, то $A(G)=\mathbb{Z}_{2}^{n} \times \mathbb{R}^{n}$. Смысл перехода от $G$ к $A(G)$ состоит в том, что при некоторых естественных условиях (например, если группа $F$ конечна; см. п. 4 далее) точная последовательность $0 \rightarrow \mathbb{R}^{n} \rightarrow A(G) \rightarrow F \rightarrow 1$ расшепляется, в то время как аналогичная последовательность $0 \rightarrow \mathbb{Z}^{n} \rightarrow G \rightarrow F \rightarrow 1$, как правило, не расщепляется.

В основе доказательства тихоновости широкого класса групп лежит 
ЛЕмма 2. Пусть $H$ - нормальная подгруппа в группе $G$, которая вместе $c$ nодгруппой $F \subset G$ порождает $G$, т.е. $G=F H$. Предположим, что

1) группь $F$ и $Н$ тихоновские;

2) любая $G$-орбита в $X(H)$ либо тривиальна, либо неограничена.

Тогда группа $G$ является тихоновской.

ДокАЗАТЕЛЬСТво (ср. с [3, лемма 2.9] или [1, лемма 4.2]). Пусть $G$ линейно действует на конусе $K$ с компактной базой $B$, лежащем в линейном топологическом пространстве $L$. Ввиду тихоновости $H$ найдется мультипликативный характер $\chi: H \rightarrow \mathbb{R}^{*}$, которому отвечает непустой выпукльй подконус $K_{\chi}=\{x \in K: h(x)=\chi(h) x, h \in H\}$. Заметим, что $h g(x)=g g^{-1} h g(x)=\chi^{g}(h) g(x)$, где $\chi^{g}(h)=\chi\left(g^{-1} h g\right), h \in H$, т.е. $g\left(K_{\chi}\right)=K_{\chi^{g}}$. Пусть $\Phi: L \rightarrow \mathbb{R}$ - линейный непрерьвньй функционал, для которого $B=K \cap \Phi^{-1}(1)$. Тогда для фиксированного $h \in H$ в силу компактности $B$ равномерно по всем $g \in G$ имеем

$$
\chi^{g}(h)=\frac{\Phi(h g(x))}{\Phi(g(x))} \leqslant \sup _{y \in B} \Phi(h y)=c(h)<\infty .
$$

Отсюда следует, что орбита $G(\log (\chi))=\log (G(\chi))$ адлитивного характера $\log (\chi)$ в $X(H)$ ограничена и поэтому тривиальна. Стало быть, $G$ оставляет характер $\chi$ неподвижным, а конус $K_{\chi}$ инвариантным. Поскольку группа $F$ тихоновская, в $K_{\chi}$ найдется $F$-инвариантный луч. Очевидно, что он будет и $G$-инвариантным.

ЗАмЕчАнИЕ. Из леммы 2 следует, что даже нормальная подгруппа в тихоновской группе не обязана являться тихоновской. В самом деле, пусть $\Gamma=\left(\mathbb{Z}_{2}(\sigma) \times \mathbb{Z}(a)\right) \cdot \mathbb{Z}^{2}$, где действие $\sigma$ представлено матрицей $\left(\begin{array}{cc}-1 & 0 \\ 0 & -1\end{array}\right)$, а действие $a$ - матрицей $\left(\begin{array}{ll}2 & 1 \\ 1 & 1\end{array}\right)$. Ясно, что ${ }^{n} \Gamma=\mathbb{Z}^{2}, F=\Gamma /{ }^{n} \Gamma=\mathbb{Z}_{2}(\sigma) \times \mathbb{Z}(a)$ и $F$-орбита любого нетривиального характера из $X\left(\mathbb{Z}^{2}\right)=\mathbb{R}^{2}$ неограничена. Поэтому $\Gamma$ является тихоновской группой с нетихоновской нормальной подгруппой $\mathbb{Z}(\sigma) \cdot \mathbb{Z}^{2}$ (она эпиморфно отображается на $\mathbb{Z}_{2} \cdot \mathbb{Z}$ ).

Заметим, что типичным примером линейного оператора, все нетривиальные орбиты которого неограничены, является унипотентньй оператор $A$ на $\mathbb{R}^{n}$ (унипотентность означает существование $l \in \mathbb{N}$ такого, что $(A-E)^{l} \equiv 0$, где $E$ и 0 - соответственно единичньй и нулевой операторы на $\left.\mathbb{R}^{n}\right)$. Это позволяет применить лемму 2 к доказательству следующего утверждения.

СлЕДСТВИЕ. Конечно порохденные нильпотентные группь явяяются тихоновскими.

ДокАЗАТЕЛьство. В самом деле, пусть $1=\Gamma_{0} \subset \Gamma_{1} \subset \cdots \subset \Gamma_{k}=\Gamma$ - цепочка нормальных подгрупп в $Г$ такая, что каждая факторгруппа $\Gamma_{l} / \Gamma_{l-1}$ циклическая и $\left[\Gamma_{l}, \Gamma_{l-1}\right] \subset \Gamma_{l-2}$. Предположим, что все $\Gamma_{i}, i \leqslant l-1$, тихоновские. Пусть $\Gamma_{l}$ порождена $\Gamma_{l-1}$ и элементом $a \in \Gamma_{l}$. Тогда действие оператора $A$ на $X\left(\Gamma_{l-1}\right)$, заданное формулой $A(\chi)(g)=\chi^{a}(g)=\chi\left(a^{-1} g a\right), g \in \Gamma_{l-1}$, является унипотентным. В самом деле, $(A-E)(\chi)(g)=\chi\left(\left[a^{-1}, g\right]\right)$, поэтому найдется $n \leqslant l$ такое, что $(A-E)^{n} \equiv 0$. Следовательно, каждая $\Gamma_{l}$-орбита в $X\left(\Gamma_{l-1}\right)$ либо тривиальна, либо неограничена, и мы можем применить лемму 2.

Теперь мы готовы доказать критерий тихоновости для почти абелевых групп. 
Лемма 3. Пусть $F$ - конечно порохденная почти абелева группа. Тогда следующие условия әквивалентны:

1) $F$ является тихоновской;

2) $F$ не допускает гомоморфизма $\varphi: F \rightarrow \mathrm{O}(n, \mathbb{R}) \cdot \mathbb{R}^{n}$ такого, что замыкание $G=\overline{\varphi(F)}$ пересекается $c \mathbb{R}^{n}$ по равномерной подгруппе и не лежит иеликом в $\mathbb{R}^{n}$

3) иентр $Z(F)$ имеет конечный индекс в $F$.

ДокАЗАтЕЛьСтво. 1) $\Longrightarrow 2$ ) следует из леммы 1. Докажем 3$) \Longrightarrow 1)$. Центр $Z(F)$ является абелевой и, следовательно, тихоновской группой. Поэтому если у нас есть $F$-инвариантньй конус $K$ в пространстве $L$, то в нем есть непустой подконус $K_{\chi}=\{x \in K: z(x)=\chi(z) x, z \in Z(F)\}$, отвечающий некоторому мультипликативному характеру $\chi: Z(F) \rightarrow \mathbb{R}^{*}$. Рассмотрим группу $\operatorname{Aut}\left(K_{\chi}\right)$ всех линейных преобразований конуса $K_{\chi}$. Она содержит в себе центральную подгруппу $D$ всех гомотетий. Так как группа $Z(F)$ центральна в $F$, характер $\chi$ является $F$-неподвижным, а конус $K_{\chi}$ является $F$-инвариантным. Пусть $\varphi: F \rightarrow \operatorname{Aut}\left(K_{\chi}\right)$ - построенное представление группы $F$. Тогда $\varphi(Z(F)) \subset D$, и, следовательно, группа $\varphi(F) D / D$ конечна. Так как группа $D$ изоморфна $\mathbb{R}$, имеет место расшепление $\varphi(F) D=C \times D$, где $C$ - некоторая конечная подгруппа в $\operatorname{Aut}\left(K_{\chi}\right)$. Пусть $x \in K_{\chi}$ - неподвижная точка относительно $C$. Тогда $\mathbb{R}^{+} x$ - искомьй $F$-инвариантный луч.

Докажем теперь 2 ) $\Longrightarrow 3$ ). В конечно порожденной почти абелевой групше всегда можно найти нормальную подгруппу конечного индекса без кручения. Пусть $\mathbb{Z}^{n}-$ такая нормальная подгруппа в $F$. Вложим $F$ в группу $A(F)$, полученную взятием линейной оболочки $\mathbb{Z}^{n} \subset F$. Так как группа $C=A(F) / \mathbb{R}^{n}=F / \mathbb{Z}^{n}$ конечна, имеет место расщепление $A(F)=C \cdot \mathbb{R}^{n}$. Пусть $C^{\prime} \subset C$ - ядро действия $C$ на $\mathbb{R}^{n}$. Тогда образ $F^{\prime} \subset\left(C / C^{\prime}\right) \cdot \mathbb{R}^{n}$ группы $F$ обладает тем свойством, что содержит $\mathbb{Z}^{n}$ и $F^{\prime} \mathbb{R}^{n}=\left(C / C^{\prime}\right) \cdot \mathbb{R}^{n}$. Отсюда по условию 2$) \quad C / C^{\prime}=1$, т.е. $A(F)=C \times \mathbb{R}^{n}$. Следовательно, $\mathbb{Z}^{n}$ лежит в центре $F$.

ЗАмЕчАнИЕ. Известно [7], что для конечно порожденных групп

$$
|G / Z(G)|<\infty \Longleftrightarrow|[G, G]|<\infty
$$

Поэтому конечность коммутанта также является критерием тихоновости для почти абелевых групп.

Приведем несколько примеров почти абелевых тихоновских групп. Пусть $F=\mathbb{Z}_{2}(\sigma)$. $\left(\mathbb{Z}_{2}(a) \times \mathbb{Z}(b)\right)$, где $\sigma(a, 1)=(a, 1)$ и $\sigma(1, b)=(1, b)$. Тогда $Z(F)=\mathbb{Z}_{2}(a) \times \mathbb{Z}\left(b^{2}\right)$ подгруппа индекса 4 в $F$. В этом примере $F$ является произведением двух нормальных подгрупп: конечной $\mathbb{Z}_{2}(\sigma) \times \mathbb{Z}_{2}(a)$ и абелевой $\mathbb{Z}_{2}(a) \times \mathbb{Z}(b)$. Легко видеть, что любая группа вида $F=K A$, где $K$ - конечная нормальная подгруппа и $A$ - абелева нормальная подгруппа, является тихоновской. Однако, такими группами не исчерпьваются все почти абелевы тихоновские группы. Пусть, например, $F=\mathbb{Z}(\sigma) \cdot \mathbb{Z}_{3}(a)$, где $\sigma(a)=a^{2}$. Тогда $Z(F)=\mathbb{Z}\left(\sigma^{2}\right)$ - подгруппа индекса 6 в $F$. Пусть, наконец, $N$ - нильпотентная группа Гейзенберга с образующими $a, b, c$ и соотношениями $[a, b]=c,[a, c]=[b, c]=1$. Положим $F=N / \mathbb{Z}\left(c^{2}\right)$. Тогда $Z(F)=\mathbb{Z}\left(a^{2}\right) \times \mathbb{Z}\left(b^{2}\right) \times \mathbb{Z}_{2}(c)$ - подгрупша индекса 4 в нильпотентной группе $F$.

Итак, если $Г$ - полициклическая группа, то ее нильрадикал ${ }^{n} \Gamma$ является тихоновской группой, а для факторгруппы $F=\Gamma{ }^{n} \Gamma$ мы только что нашли критерий тихоновости. 
В дальнейшем нам понадобится следуюшее замечание о векторном пространстве непрерьвных аддитивных характеров. Отметим, что действие $G$ сопряжениями на $X(G)$ тривиально. Пусть $H$ является нормальной подгруппой в группе $G$ и $F=G / H$. Обозначим через $\xi: X(G) \rightarrow X(H)$ функтор ограничения. Пусть $X(H, G)=\operatorname{Im}(\xi)-$ пространство характеров на $H$, которые могут быть продолжены до характеров $G$. Ясно, что $\operatorname{Ker}(\xi)$ изоморфен $X(F)$, т.е. $X(G) / X(F) \simeq X(H, G)$. Предположим, что автоморфизм $\sigma$ групшы $G$ оставляет подгруппу $H$ инвариантной. Действие $\sigma$ на $X(G)$, заданное формулой $\chi^{\sigma}(g)=\chi(\sigma(g))$, индуцирует действия на $X(F)$ и $X(H, G)$. Поэтому если автоморфизм $\sigma$ тривиален на $X(F)$ и $X(H, G)$, то он унипотентен на $X(G)$.

Докажем теперь критерий тихоновости для полициклических групш.

ТеОремА 1. Пусть Г-полиииклическая группа с нильрадикалом ${ }^{n} \Gamma$. Тогда следующие условия әквивалентны:

1) Г является тихоновской группой;

2) $Г$ не допускает гомоморфизма $\varphi$ в группу вида $\mathrm{O}(n, \mathbb{R}) \cdot \mathbb{R}^{n}$ такого, что замыкание $\overline{\varphi(\Gamma)}$ пересекается $c \mathbb{R}^{n}$ по равномерной подгруппе и не лежит иеликом в $\mathbb{R}^{n}$;

3) чентр почти абелевой факторгруппь $F=\Gamma /{ }^{n} \Gamma$ имеет конечный индекс в $F$, и любая $F$-орбита в векторном пространстве $X\left({ }^{n} \Gamma\right)$ аддитивных характеров ${ }^{n}$ Г либо тривиальна, либо неограничена.

ДокАЗАТЕЛЬСтво. 1) $\Longrightarrow 2$ ) следует из леммы 1.

Докажем 3 ) $\Longrightarrow 1$ ). Пусть $K$ - вьпуклый конус с компактной базой и заданньм на нем линейным действием группы $G$. Так как нильрадикал ${ }^{n} \Gamma$ является тихоновской группой, найдется мультипликативньй характер $\chi_{0}:{ }^{n} \Gamma \rightarrow \mathbb{R}^{*}$, которому соответствует непустой подконус $K_{0}=\left\{x \in K: h(x)=\chi_{0}(h) x, h \in{ }^{n} \Gamma\right\}$. Поскольку Г-орбита $\log \left(\chi_{0}\right) \in X\left({ }^{n} \Gamma\right)$ не может быть неограниченной ввиду компактности базы конуса $K$, она является тривиальной, поэтому Г оставляет $\chi_{0}$ неподвижным, а $K_{0}$ инвариантным. Рассмотрим возрастающую последовательность подгрупп $1=F_{0} \subset \cdots \subset F_{k}=Z(F)$ такую, что каждая факторгруппа $F_{l} / F_{l-1}$ циклическая. Пусть $p: \Gamma \rightarrow F$ - проекция вдоль ${ }^{n} \Gamma, H=p^{-1}(Z(F)), H_{l}=p^{-1}\left(F_{l}\right) \subset H$. Докажем индукцией по $l=0, \ldots, k$, что $H$ имеет инвариантньй луч в $K_{0}$. В самом деле, пусть найден характер $\chi_{l}: H_{l} \rightarrow \mathbb{R}^{*}$, которому соответствует нетривиальньй подконус $K_{l}=\left\{x \in K: h(x)=\chi_{l}(h) x, h \in H_{l}\right\}$. Возьмем элемент $a \in H_{l+1}$, которьй вместе с $H_{l}$ порождает $H_{l+1}$. Известно, что автоморфизм пространства $X\left(H_{l}\right)$, заданньй сопряжением элементом $a$, тривиально действует на подпространстве $X\left(F_{l}\right)$ (так как $\left[H_{l+1}, H_{l}\right] \subset{ }^{n} \Gamma$ ) и оставляет неподвижной проекцию $\log \left(\chi_{0}\right) \in X\left({ }^{n} \Gamma, H_{l}\right)$ характера $\log \left(\chi_{l}\right)$ вдоль $X\left(F_{l}\right)$. Следовательно, $a$-орбита характера $\log \left(\chi_{l}\right) \in X\left(H_{l}\right)$ является унипотентной, поэтому ввиду компактности базы конуса $K_{l}$ она является тривиальной. Это означает, что элемент $a \in H_{l+1}$ оставляет конус $K_{l}$ инвариантным, значит $H_{l+1}$ имеет в $K_{l}$ инвариантный луч.

Итак, мы нашли нетривиальный подконус $K_{\eta}=\{x \in K: h(x)=\eta(h) x, h \in H\}$, соответствующий некоторому характеру $\eta: H \rightarrow \mathbb{R}^{*},\left.\eta\right|_{\Gamma} \equiv \chi_{0}$. Заметим, что каждый элемент $a \in$ Г оставляет $\eta$ неподвижным. В самом деле, ограничение $\log (\eta)$ на ${ }^{n} \Gamma$ совпадает с $\log \left(\chi_{0}\right)$ и, следовательно, является $\Gamma$-неподвижньм, а действие $\Gamma$ на $X(Z(F))$ тривиально (так как ${ }^{n} \Gamma$ тривиально действует на $X(H)$, а $F=\Gamma /{ }^{n} \Gamma-$ на $X(Z(F)) \subset X(H)$, причем $\left.X(H) / X(Z(F))=X\left({ }^{n} \Gamma, H\right)\right)$. Таким образом, $a$-орбита характера $\log (\eta)$ является унипотентной и ввиду компактности базы конуса $K_{0}$ тривиальной. Следовательно, Г оставляет характер $\eta$ неподвижным, а конус $K_{\eta}$ инвариантным. Заметим, что $H$ 
действует на $K_{\eta}$ гомотетиями, а факторгрупа $\Gamma / H$ конечна. Используя теперь тот же прием расщепления, что и в лемме 3 , мы найдем $\Gamma$-инвариантный луч в $K$.

Докажем 2) $\Longrightarrow 3$ ). Если группа Г удовлетворяет условию 2), то и ее почти абелева факторгруппа $F=\Gamma /{ }^{n} \Gamma$ также удовлетворяет условию 2 ) и по лемме 3 является тихоновской. Рассмотрим разложение ${ }^{n} \Gamma /\left[{ }^{n} \Gamma,{ }^{n} \Gamma\right]=\mathbb{Z}^{n} \times$ Tor в свободную абелеву групшу и кручение. Так как любой характер тривиален на коммутанте и всех конечных подгруппах, имеет место $X\left({ }^{n} \Gamma\right) \simeq X\left(\mathbb{Z}^{n}\right)=\mathbb{R}^{n}$. Не ограничивая общности (переходя, если необходимо, от $\Gamma$ к $\left(\Gamma /\left[{ }^{n} \Gamma,{ }^{n} \Gamma\right]\right) /$ Tor $)$, можно считать, что ${ }^{n} \Gamma=\mathbb{Z}^{n}$. Итак, $\mathbb{Z}^{n}$ является нильрадикалом в $\Gamma$, и центр групш $F=\Gamma / \mathbb{Z}^{n}$ имеет конечный индекс в $F$. Рассмотрим действие группы $F$ на векторном пространстве $X\left(\mathbb{Z}^{n}\right) \simeq \mathbb{R}^{n}$.

Лемма 4. Пусть группа $F$, чентр которой имеет конечный индекс в $F$, действует на $\mathbb{R}^{n}$. Тогда либо

1) любая F-орбита в $\mathbb{R}^{n}$ тривиальна или неограничена, либо

2) найдется $F$-инвариантное подпространство $V \subset \mathbb{R}^{n}$ такое, что образ $F$ в $\mathrm{GL}\left(\mathbb{R}^{n} / V\right)$ предкомпактен и нетривиален.

ДокАЗАТЕЛЬСтво. Неограничивая общности, можнопредположить, что $\mathbb{R}^{n}$ не раскладьвается в сумму собственных $F$-инвариантных подпространств. Рассмотрим естественньй гомоморфизм $\varphi: F \rightarrow \operatorname{GL}(n, \mathbb{R})$ и возьмем замыкание $P$ по Зарисскому подгруппы $\varphi(F) \subset \mathrm{GL}(n, \mathbb{R})$. Тогда $Z(P)$ имеет конечньй индекс в $P$ и потому $P=D \times U \times K$, где $K$ компактна (и центр $Z(K)$ имеет конечньй индекс в $K$ ), $D$ диагонализируема, $U$ абелева и унипотентна. Разложим $\mathbb{R}^{n}$ в сумму корневых подпространств относительно $D$. Тогда $\mathbb{R}^{n}=V+V^{\prime}$, где $V$ состоит из всех $D$-неподвижньх векторов в $\mathbb{R}^{n}$, а $V^{\prime}$ является суммой всех нетривиальных корневых подпространств. Так как $D$ нормальна в $P$, оба подпространства $P$-инвариантны, и ввиду неразложимости либо $V=0$, либо $V^{\prime}=0$.

В первом случае все $P$ - и $F$-орбиты в $\mathbb{R}^{n}$ неограничены (так как $D$ действует на любом корневом подпространстве из $V^{\prime}$ гомотетиями). Во втором случае группа $D$ тривиальна. Рассмотрим фильтрацию $\mathbb{R}^{n}$ относительно действия унипотентной подгруппы $U$ :

$$
0=V_{0} \subset V_{1} \subset \cdots \subset V_{k}=\mathbb{R}^{n}, \quad \text { где } V_{l} / V_{l-1}=\left\{x \in \mathbb{R}^{n} / V_{l-1}: U(x)=x\right\} .
$$

Так как группа $U$ нормальна в $P$, каждоеподпространство $V_{l}$ является $P$-инвариантным. Если действие компактной группы $K$ на факторпространстве $\mathbb{R}^{n} / V_{k-1}$ нетривиально, что соответствует утверждению 2) леммы. Если же оно тривиально, то из полной приводимости действия $K$ на $\mathbb{R}^{n}$ следует, что подпространство $K$-неподвижных векторов в $\mathbb{R}^{n}$ нетривиально. Из полной приводимости $K$, нормальности $K$ в $P$ и неразложимости $\mathbb{R}^{n}$ следует, что групша $K$ тривиальна, значит группы $P$ и $\varphi(F)$ унипотентны, что соответствует утверждению 1) леммы.

ЗАмечАниЕ. Для произвольных почти абелевых груп лемма неверна. Пусть, например, $F=\mathbb{Z}_{2}(\sigma) \cdot \mathbb{Z}(a)$, где $\sigma=\left(\begin{array}{cc}-1 & 0 \\ 0 & 1\end{array}\right), a=\left(\begin{array}{ll}1 & 1 \\ 0 & 1\end{array}\right)$. Тогда если $e_{1}=(1,0)$, то $F\left(e_{1}\right)= \pm e_{1}$, а $F$-орбита любого вектора вне $\mathbb{R} e_{1}$ неограничена. В то же время действие $F$ на единственном факторпространстве $\mathbb{R}^{2} / \mathbb{R} e_{1}$ тривиально.

Закончим доказательство теоремы 1. Итак, осталось доказать, что случай 2) из леммы 4 невозможен при условии 2) теоремы 1. Рассмотрим вложение $Г$ в группу $A(\Gamma)$, полученную взятием линейной оболочки нильрадикала $\mathbb{Z}^{n} \subset \Gamma$. Тогда $\mathbb{R}^{n}$ является нормальной подгруппой в $A(\Gamma), \Gamma \cap \mathbb{R}^{n}=\mathbb{Z}^{n}$ и $\Gamma \mathbb{R}^{n}=A(\Gamma)$. Ясно, что орбиты $F$ на $\mathbb{R}^{n}$ 
суть орбиты $F$ на $X\left(\mathbb{Z}^{n}\right)$. Допустим, что внутри $\mathbb{R}^{n}$ нашлась хотя бы одна нетривиальная ограниченная $F$-орбита. Используя лемму 4 и переходя, если необходимо, к факторгруппе $A(\Gamma) / V$ со взятием замыкания образа Г в этой факторгруппе, мы можем считать, что образ $F$ в $\mathrm{GL}(n, \mathbb{R})$ предкомпактен и нетривиален. Пусть $\varphi: F \rightarrow \operatorname{GL}(n, \mathbb{R})$ - естественное представление. Так как $\overline{\varphi(F)}$ - компакт в $\mathrm{GL}(n, \mathbb{R}), F$-действие на $\mathbb{R}^{n}$ вполне приводимо. Следовательно, не ограничивая общности, можно считать, что оно неприводимо. Теперь, чтобы прийти к противоречию с условием 2) теоремы 1 , осталось доказать следующее утверждение.

Лемма 5. Пусть $\mathbb{R}^{n}$ - нормальная подгруппа в $G, F=G / \mathbb{R}^{n} u \varphi: F \rightarrow \operatorname{GL}(n, \mathbb{R})$ индуцированное представление. Предположим, что $\varphi(F) \subset \mathrm{GL}(n, \mathbb{R})$ - нетривиальная предкомпактная группа с неприводимым действием на $\mathbb{R}^{n}$ и иентр $Z(F)$ имеет конечный индекс в $F$. Тогда $G$ допускает эпиморфизм на расщепимую груп$n y \varphi(F) \cdot \mathbb{R}^{n}$.

ДокАЗАТЕЛЬСТво. Пусть $Z(F)=\mathbb{Z}^{m} \times T$ - разложение в свободную абелеву группу и кручение, $p: A(\Gamma) \rightarrow F$ - естественная проекция. Поскольку действие $F$ на $\mathbb{R}^{n}$ неприводимо, для любого элемента $a \in Z(F)$ его действие на $\mathbb{R}^{n}$ либо тривиально, либо не имеет неподвижных точек. Итак, возможны только два случая:

1) $\mathbb{Z}^{m}$ тривиально действует на $\mathbb{R}^{n}$;

2) хотя бы один элемент $a \in \mathbb{Z}^{m}$ не имеет неподвижных точек в $\mathbb{R}^{n}$.

В первом случае нормальная подгруппа $H=p^{-1}\left(\mathbb{Z}^{m}\right) \subset A(\Gamma)$ нильпотентна, а действие конечной групшы $K=F / \mathbb{Z}^{m}$ на $\mathbb{R}^{n}$ ввиду $F$-неприводимости не имеет неподвижных точек. Докажем, что группа $H$ абелева, т.е. $H=\mathbb{Z}^{m} \times \mathbb{R}^{n}$. В самом деле, пусть $i: H \rightarrow N$ - вложение $H$ в качестве равномерной подгрупшы в связную односвязную нильпотентную групу Ли $N, N / \mathbb{R}^{n}=\mathbb{R}^{m}$. Подобно тому, как мы брали линейную оболочку нормальной подгрупшы $\mathbb{Z}^{n} \subset G$, можно рассмотреть групу $G^{*}$, полученную из $G$ взятием лиевой оболочки $N$ нильпотентной групшы $H$. А именно, $G^{*}=(G \cdot N) / \Delta_{H}$, где полупрямое произведение получено из продолжения $G$-действия с $H$ на $N$, а $\Delta_{H}=\left\{\left(h, i(h)^{-1}\right): h \in H\right\}$ - “антидиагональ" в $H \cdot i(H)$.

Тогда можно считать, что $G^{*} \cap N=H, N$ - нормальная подгруппа в $G^{*}$ и $G^{*} / N=$ $G / H=F / \mathbb{Z}^{m}=K$. Так как $N$ - односвязная нильпотентная группа и $K$ конечна, имеет место расщепление $G^{*}=K \cdot N$ (см. п. 4 ниже). Пусть $Z_{N}(K)$ - централизатор $K$ в $N$. Так как $K$ на $N / \mathbb{R}^{n}$ действует тривиально и действие $K$ на $N$ вполне приводимо, выполнено $N=Z_{N}(K) \mathbb{R}^{n}$. Но $Z_{N}(K) \cap \mathbb{R}^{n}=0$, поэтому имеет место расщепление $N=Z_{N}(K) \cdot \mathbb{R}^{n}$, причем группа $Z_{N}(K)$ изоморфна $\mathbb{R}^{m}$. Теперь ввиду нильпотентности $N=\mathbb{R}^{m} \times \mathbb{R}^{n}$ и $H=\mathbb{Z}^{m} \times \mathbb{R}^{n}$. Так как абелева групша $N$ нормальна в $G^{*}$ и действие конечной группы $K=G^{*} / N$ на $N / \mathbb{R}^{n}=\mathbb{R}^{m}$ тривиально, $\mathbb{R}^{m}$ лежит в центре $G^{*}$, а $\mathbb{Z}^{m}$ в центре $A(\Gamma)$. Ввиду конечности $K$ имеет место расщепление $G / \mathbb{Z}^{m}=K \cdot \mathbb{R}^{n}$, т.е. $G$ проектируется на групу $\varphi(F) \cdot \mathbb{R}^{n}$, где $\varphi(F)$ - факторгруппа $K$.

Во втором случае возьмем элемент $g \in A(G)$ такой, что $p(g)=a$. Поскольку $а$ лежит в центре $F$ и не имеет неподвижных точек на $\mathbb{R}^{n}$, имеет место расщепление $G=F \cdot \mathbb{R}^{n}$ (см. п. 4), что завершает доказательство леммы 5.

Теорема 1 также доказана.

ЗАмЕчАниЕ. Вообще говоря, если $\mathbb{R}^{n}$ - нормальная подгруппа в $G$ и $F=G / \mathbb{R}^{n}-$ почти абелева группа с неприводимьм ограниченным действием на $\mathbb{R}^{n}$, группа $G$ не обязана допускать эпиморфизм на расщепимую групу $\varphi(F) \cdot \mathbb{R}^{n}$. Пример: $G=Z_{2}(\sigma) \cdot N$, где $N$ - нильпотентная группа Гейзенберга с линейной оболочкой ее центра (т.е. 
$Z(N)=\mathbb{R} c$ - центр в $N, N / Z(N)=\mathbb{Z}(a) \times \mathbb{Z}(b),[a, b]=c)$, причем $\sigma(a)=b, \sigma(b)=a$, $\sigma(c)=-c$. Тогда $F=G / \mathbb{R} c=\mathbb{Z}_{2}(\sigma) \cdot(\mathbb{Z}(a) \times \mathbb{Z}(b))$ - почти абелева группа с неприводимым ограниченным действием на $\mathbb{R} c$, однако $G$ не допускает эпиморфизма на $\varphi(F) \cdot \mathbb{R} c=\mathbb{Z}_{2}(\sigma) \cdot \mathbb{R} c$.

ПРимеР. Вообще говоря, неверно, что если полициклическая группа не является тихоновской, то она допускает эпиморфизм на группу вида $C \cdot \mathbb{Z}^{n}$, где $C$-нетривиальная конечная подгруппа в $\mathrm{SL}(n, \mathbb{Z})$. В самом деле, пусть

$$
a=\left(\begin{array}{cccc}
0 & 1 & 0 & 0 \\
0 & 0 & 1 & 0 \\
0 & 0 & 0 & 1 \\
-1 & 8 & -10 & 8
\end{array}\right)
$$

Тогда $a$-действие на $\mathbb{R}^{4}$ задает разложение $\mathbb{R}^{4}=V+W$ в сумму двух $a$-инвариантных плоскостей, причем

1) обе плоскости имеют плотньй образ в торе $\mathbb{R}^{4} / \mathbb{Z}^{4}$;

2) $a$ действует на $V$ ортогональньм поворотом на угол несоизмеримый с $2 \pi$;

3) а действует на $W$ гиперболическим поворотом.

Таким образом, $\Gamma=\mathbb{Z}(a) \cdot \mathbb{Z}^{4}$ допускает гомоморфизм в групш $\mathrm{SO}(2, \mathbb{R}) \cdot V$ с плотньм образом и, значит, не является тихоновской. В то же время единственной нетривиальной факторгруппой $\Gamma$ является $\mathbb{Z}(a)=\Gamma / \mathbb{Z}^{4}$. Заметим также, что $\Gamma$ не содержит тихоновских подгруш конечного индекса.

3. Тихоновское свойство для линейных групп и групп Ли. Единственный факт о строении полициклических груп, которьй нам потребовался, - это почти абелевость факторгрупшы $F=\Gamma /^{n} \Gamma$. Ясно, что таким же свойством обладают почти полициклические группы. Известно (альтернатива Титса [4]), что любая линейная дискретная группа либо содержит свободную группу $F_{2}$ (и поэтому не является даже аменабельной), либо является почти полициклической. Таким образом, мы установили критерий тихоновости для дискретных линейных групп.

С другой стороны, связная группа Ли $G$ аменабельна тогда и только тогда, когда $G=K R$, где $R$ - радикал в $G$ и $K$ - компактная полупростая подгруппа. Пусть $N-$ нильрадикал в $G$. Тогда радикал $A=R / N$ факторгрупшы $F=G / N$ является центральной связной подгруппой в $F=K A$, причем $K$ компактна и нормальна в $F$. Поэтому $N$ и $F$ являются тихоновскими (доказательство для $F$ идентично доказательству $2) \Longrightarrow 3$ в лемме 3 ; достаточно заменить циклические подгрупы на однопараметрические). Ясно, что если $G$ допускает гомоморфизм с плотным образом в группу вида $C \cdot \mathbb{R}^{n}$, где $C$ - нетривиальная компактная подгруппа в $\mathrm{O}(n, \mathbb{R})$, то $G$ не является тихоновской.

Если такого гомоморфизма нет, рассмотрим действие связной групшы $F=K A$ на векторном пространстве $X(N)$. Как и при доказательстве 2$) \Longrightarrow 3$ ) в теореме 1 , мы найдем $G$-инвариантный луч для каждого линейного $G$-действия на вьпуклом конусе с компактной базой. Мы доказали критерий тихоновости для групп Ли, которьй по существу совпадает с критерием из [2].

ТЕОремА 2. Пусть $G$ - связная аменабельная группа Ли с нильрадикалом $N$. Тогда следующие условия әквивалентны:

1) группа $G$ тихоновская;

2) $G$ не допускает гомоморфизма с плотным образом в полупрямое произведение вида $C \cdot \mathbb{R}^{n}$, әде подгруппа $C \subset \mathrm{O}(n, \mathbb{R})$ нетривиальна; 
3) любая нетривиальная $G$-орбита на векторном пространстве $X(N)$ непрерывных аддитивных характеров $N$ неограничена.

4. Приложение. В доказательстве основной теоремы мы использовали следующие несложные факты о расшепляемости групш.

ПРЕДЛОЖЕНИЕ 1. Если $\mathbb{R}^{n}$ - нормальная подгруппа в $G$ и факторгруппа $F=$ $G / \mathbb{R}^{n}$ компактна, то $G$ изоморфна полупрямому произведению $F \cdot \mathbb{R}^{n}$.

ДокАЗАТЕЛьСтво. Поскольку $\mathbb{R}^{n}$ - абелева нормальная подгруппа в $G$, внутренние автоморфизмы $G$ порождают действие $\rho$ групшы $F$ на $\mathbb{R}^{n}$. Известно [7], что препятствие к расшепляемости точной последовательности $0 \rightarrow \mathbb{R}^{n} \rightarrow G \rightarrow F \rightarrow 1$ лежит во второй группе когомологий $H^{2}\left(F, \rho, \mathbb{R}^{n}\right)$. Однако, все групшы когомологий действия компактной групш на $\mathbb{R}^{n}$ тривиальны, поэтому никаких препятствий к расщепляемости нет.

ЗАмЕчАниЕ. Ясно, что в предложении 1 нельзя заменить $\mathbb{R}^{n}$ на $\mathbb{Z}^{n}$, так как последовательность $0 \rightarrow 2 \mathbb{Z} \rightarrow \mathbb{Z} \rightarrow \mathbb{Z}_{2} \rightarrow 1$ не расщепляется. $\mathrm{C}$ другой стороны, $\mathbb{R}^{n}$ можно заменить любой связной односвязной нильпотентной группой Ли $N$. Это легко доказывается индукцией по длине нильпотентности $N$.

ПРЕДЛОЖЕНИЕ 2. Пусть $\mathbb{R}^{n}$ - нормальная подгруппа в $G, F=G / \mathbb{R}^{n}$ up: $G \rightarrow F$ - естественная проекиия. Допустим, что действие әлемента $а \in G$ на $\mathbb{R}^{n}$ не имеет неподвижных векторов и $p(a)$ лежит в иентре $F$. Тогда имеет место расщепление $G=Z_{G}(a) \cdot \mathbb{R}^{n}$.

ДокАЗАтЕльство. Достаточно доказать, что для всякого $f \in F$ найдется $g \in G$ такой, что $p(g)=f$ и $g$ коммутирует с $a$. Тогда мы докажем, что $p\left(Z_{G}(a)\right)=F$ и, следовательно, $G=Z_{G}(a) \mathbb{R}^{n}$. Так как $Z_{G}(a) \cap \mathbb{R}^{n}=0$, получим искомое расщепление.

Пусть $p(h)=f$. Тогда $a h a^{-1}=h x$ для некоторого $x \in \mathbb{R}^{n}$. Найдем $y \in \mathbb{R}^{n}$ такой, что $a(h y) a^{-1}=h y$. Так как $a(h y) a^{-1}=a h a^{-1} a y a^{-1}=h x\left(a y a^{-1}\right)$, достаточно решить уравнение $a y a^{-1} y^{-1}=x^{-1}$ относительно $y \in \mathbb{R}^{n}$. В аддитивной записи это уравнение имеет вид $(A-E) y=-x$, где $A(y)=a y a^{-1}$. Так как оператор $A$ на $\mathbb{R}^{n}$ не имеет неподвижных векторов (кроме нулевого), оператор $A-E$ обратим, значит уравнение разрешимо.

Автор выражает признательность Р. И. Григорчуку за постановку проблемы и интерес к данной работе.

\section{СПИСОК ЦИТИРОВАННОЙ ЛИТЕРАТУРЫ}

[1] Furstenberg H. Translation-invariant cones of functions on semi-simple Lie groups // Bull. Amer. Math. Soc. 1965. V. 71. P. 271-326.

[2] Conze J.P., Guivarc'h Y. Proprieté de droite fixe et fonctions propres des opérateurs de convolution. Preprint: Univ. de Rennes.

[3] Grigorchuk R. I. On Tychonoff Groups. Preprint № 95-12: Max-Planck-Inst. fur Mathematik, 1995.

[4] Tits J. Free subgroups in linear groups // J. Algebra. 1972. V. 20. P. 250-270.

[5] Дэй М. М. Нормированные линейные пространства. М.: ИЛ, 1961.

[6] Робертсон А., Робертсон Б. Топологические векторные пространства. М.: Мир, 1967.

[7] Браун К. Когомологии групп. М.: Наука, 1987. 